\title{
Design Generativo e Design da Informação: uma aproximação necessária
}

\section{Generative Design and Information Design: a necessary approach}

Matheus Dantas de Moraes Almeida, Ana Carolina Kalume Maranhão

\begin{abstract}
Design Generativo, Design da Informação, Técnicas, Tecnologias
O artigo tem como objetivo a realização de uma revisão bibliográfica acerca de conceitos estruturais para o estudo das técnicas, tecnologias e marcos relacionados ao Design Generativo. Toma-se como base a aproximação com o Design da Informação pela premissa de que a disciplina pode oferecer recursos técnicos que nos ajudem a monitorar as tecnologias no Design Generativo. Propõem-se modelos de visualizações gráficas que, assistidas por sistemas computacionais, podem facilitar a observação sistemática da evolução tecnológica no tema, facilitando a tomada de decisão junto à comunidade.
\end{abstract}

Generative Design, Information Design, Techniques, Technologies

This article aims to carry out a literature review on structural concepts for the study of techniques, technologies and milestones related to Generative Design. The approach to Information Design is based on the premise that the discipline can offer technical resources that help us monitor technologies in Generative Design. Graphical visualization models are proposed that, assisted by computational systems, can facilitate the systematic observation of technological evolution in the subject, facilitating decisionmaking with the community.

\section{Introdução}

Enquanto disciplina, o Design Generativo (Generative Design) assume papel cada vez mais presente na nossa sociedade. Por estruturar-se também em sistemas computacionais, apresenta um novo contexto para o designer, que passa a atuar interagindo com Sistemas Generativos, pensados a partir de algoritmos para a resolução de problemas de design.

Apesar de não ser recente, sua relevância e aumento de interesse vêm assumindo um novo patamar, intensificado pelo barateamento e crescimento de performance decorrentes das tecnologias disponíveis na nuvem (Cloud Computing) e pela assimilação de técnicas de Aprendizado de Máquina (Machine Learning) (Harvard Business Review Analytic Services, 2018). Tal cenário amplia as possibilidades, podendo revelar novas dinâmicas tecnológicas e mercadológicas que podem causar impactos significativos na forma de fazer e pensar o design.

Segundo a consultoria americana Mckinsey (2018), em um estudo que avalia as tendências de modificações na força de trabalho, a entrada da Inteligência Artificial promete até 2030 modificar consideravelmente as dinâmicas econômicas, com projeções de aumento de $50 \%$ do

Anais do $10^{\circ} \mathrm{CIDI}$ e $10^{\circ} \mathrm{CONGIC}$

Kelli C.A.S. Smythe, Rafael de Castro Andrade (orgs.) Sociedade Brasileira de Design da Informação - SBDI Curitiba | Brasil | 2021
Proceedings of the $10^{\text {th }} \mathrm{CIDI}$ and $10^{\text {th }}$ CONGIC

Kelli C.A.S. Smythe, Rafael de Castro Andrade (orgs.)

Sociedade Brasileira de Design da Informação - SBDI Curitiba | Brazil | 2021 
tempo gasto com o uso de habilidades tecnológicas avançadas e crescimento de $19 \%$ nas demandas por habilidades cognitivas superiores, como criatividade, pensamento crítico, tomada de decisão e processamento de informações complexas.

Na perspectiva mercadológica, o estudo da McKinsey (2018) demonstra que é esperada uma transição importante nas dinâmicas de trabalho, o que nos traz à tona o questionamento sobre como o campo do Design tem se preparado para possíveis mudanças, uma vez que as quatro principais áreas com maior incompatibilidade entre habilidades e ofertas de trabalho têm grande influência do Design, como é o caso da Ciência de Dados, da Tecnologia da Informação (desenvolvimento mobile e design de interfaces web), Pesquisa e Desenvolvimento e Design de Produto ou Serviço.

Entender as tecnologias que o Design assimila pode ser um primeiro passo para o entendimento mais profundo da problemática em questão. Assim, o presente artigo tem como objetivo o levantamento de algumas das tecnologias e marcos relacionados ao Design Generativo, em uma perspectiva técnica, além de uma aproximação estratégica com o campo do Design da Informação, que pode dar recursos que permitam sistematizar visualmente o monitoramento sobre os avanços na área. Propõe-se um conjunto de visualizações gráficas que facilitem a análise e a tomada de decisão junto à comunidade. Espera-se, assim, que o trabalho estimule novas investigações com foco no Design e Tecnologia, dentro e fora da Universidade.

\section{O Design Generativo: novas situações, novos problemas}

Amplamente difundidos no Design, os sistemas computacionais que auxiliam no processo de resolução de problemas não são novidades. Atuando na criação de formas, visualizações, edições, processamento de dados e uma série de outras funções, tais sistemas permitem um conjunto de possibilidades de intervenção e/ou o estabelecimento de parâmetros para alcançar os resultados desejados.

O Design Generativo, estruturado também na utilização de sistemas computacionais, vem construindo sua narrativa há pelo menos 50 anos. Follet (2020) destaca o pioneirismo dos primeiros artistas programáticos (programmatic artists): Vera Molnár, artista húngara que em 1960 começou a aplicar linguagens de programação para gerar imagens automatizadas, Manfred Mohr, artista digital alemão que dedicou sua produção de 1960 a 1970, e a publicação "Shape Grammars and the Generative Specification of Painting and Sculpture (1972)", dos pesquisadores George Stiny e James Gips, que originou as Gramáticas da Forma (Shape Grammars), sistemas capazes de produzir e gerar formas.

Com o passar dos anos, diversas iterações têm sido aplicadas em campos como Engenharia, Arquitetura, Design de Produto e Arte, e novas aplicações tornam-se cada vez mais presentes no nosso dia a dia, como no recente lançamento do software de Design Generativo da empresa americana Autodesk ${ }^{1}$, que utiliza abordagens evolutivas encontradas

\footnotetext{
${ }^{1}$ Disponível em: <https://www.autodesk.com/solutions/generative-design>

${ }^{2}$ Disponível em: <https://www.youtube.com/watch?v=t-VUpX-xVo4\&t=375s> 
na natureza para auxiliar na criação automatizada de um número extenso de formas a partir de parâmetros configurados.

Ainda que muito associado a ferramentas e técnicas utilizadas em representações digitais, o Design Generativo não está limitado ao ambiente digital, bem como não está interessado somente na criação de produtos, mas em novas dinâmicas e processos de criação. Tal enfoque no âmbito digital, ainda assim, justifica-se por permitir análises anteriores às etapas de modelagem, produção ou aplicação (Fisher e Herr, 2001). Assim, o contexto do Design Generativo, por natureza, modifica a abordagem tradicional ao incluir um novo elemento, os Sistemas Generativos, como mostra a Figura 1.

Figura 1: Abordagem no design a partir do uso de Sistemas Generativos. Fisher e Herr (2001) adaptado pelo autor.
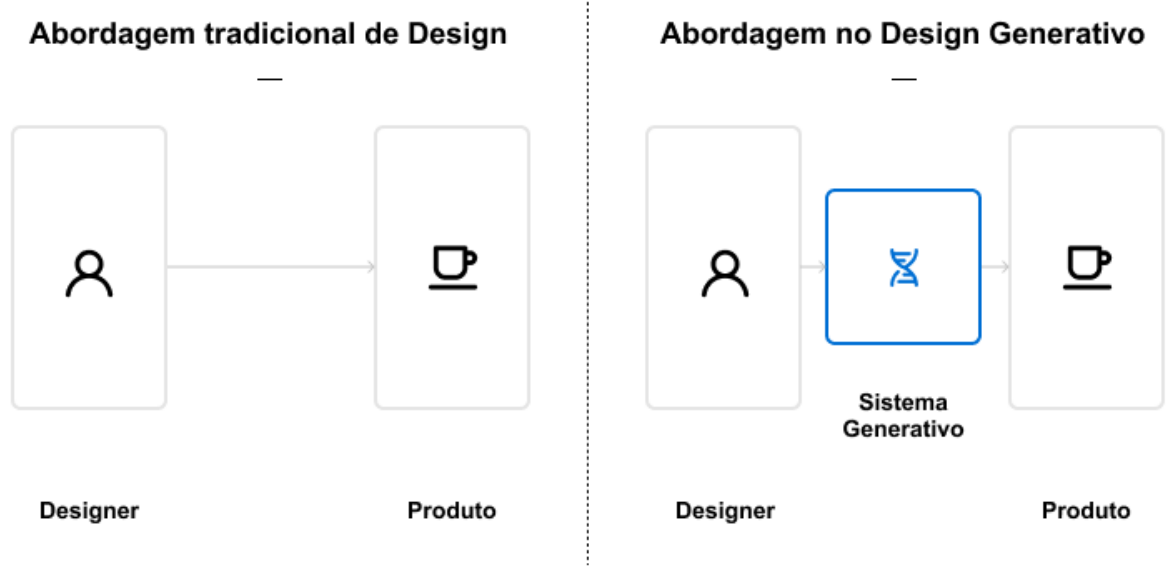

Fisher e Herr (2001) demonstram que, nos moldes tradicionais, o designer, motivado pelo problema de design, gerava produtos de design. Já na abordagem do Design Generativo, o designer passa a interagir com sistemas generativos que o auxiliam na criação das soluções, ou seja, a ação é intermediada por essa nova variável.

A partir de tal intermediação, há uma expectativa por mais produtividade, muito relacionada à quantidade de soluções possíveis geradas pelos Sistemas Generativos, além da expectativa de otimização na seleção de "bons designs". Fisher e Herr (2001) defendem que somente em problemas de design muito restritos isso é possível, mas que pela complexidade cultural, ética e estética dos principais problemas, a presença humana ainda é essencial. Questiona-se também a definição do que poderia ser considerado um "bom design", contexto amplo e dificilmente definido de forma objetiva.

Como disciplina, o Design Generativo vivencia um balanço de forças que demandam atenção. De um lado, o avanço técnico, cada vez mais acelerado, gera soluções incrementais, inovadoras e relevantes, e de outro há uma necessidade cada vez maior de compreensão e reflexão sobre os eventuais impactos dessas técnicas. Toma-se como base a perspectiva técnica, abordada na seção que segue. 


\section{Design Generativo: técnicas e classificações}

Atualmente, as técnicas utilizadas no Design Generativo geram ferramentas criadas de modo ainda bastante particular a cada problema de design. Portanto, mesmo com esforços para a construção de softwares que apliquem técnicas integradas, não há uma solução adaptável a todos os problemas, fator que faz com que os autores também sejam criadores de suas próprias ferramentas.

A defesa pela criação ativa de ferramentas, como proposto por Arida (2004), parte da necessidade de estabelecer regras que não limitem ou sacrifiquem a função e o toque do designer. Para ele, há uma necessidade de desenvolvimento de habilidades algorítmicas e um conhecimento ampliado e conceitual sobre regras.

Diante disto, a partir da visão de Fischer e Herr (2001), que mapearam um acervo de técnicas a serem ensinadas em uma disciplina de Design Generativo, e Singh e Gu (2012), que apresentam quatro conjuntos de técnicas, propõe-se uma visualização que segue na Figura 2. Considera-se a formatação hierárquica de árvore (Meirelles 2013 p. 18), que se estrutura em um dendograma subdividido em quatro conjuntos de elementos principais: i) Sistemas Emergentes; ii) Gramáticas Generativas; iii) Geração Algorítmica e crescimento; iv) (Re) Produção Algorítmica (Design Evolucionário). Entre os agrupamentos estruturam-se cada uma das técnicas especificadas pelos autores:

1. Sistemas Emergentes (Emergent Systems)

a. Modelos baseados em agentes (ABS - Agent Based Models ou Agent Based Complex Systems -ACSs) - Carley (1994); Macy \& Willer (2002); Grimm et al., (2005);

i. $\quad$ Modelagem de Ninho (Swarm Modelling) - Singh \& Gu (2012);

b. Autômato Celular (Cellular Automata) - von Newmann (1951); Wolfram (2002);

2. Gramáticas Generativas (Generative Grammars)

a. Sistemas L (L-Systems) - Lindenmayer (1968); Prusinkiewicz \& Lindenmayer, (1991)

b. Gramáticas da Forma (Shape Grammars) - Stiny (1975); Stiny (1980);

3. Geração algorítmica e crescimento (Algorithmic Generation and Growth)
a. Design Paramétrico (Parametric Design) - Frazer (2016);
b. Fractais (Fractals) - Fisher \& Herr (2001); Ediz \& Çaðdap (2007)
c. Re-writing rules - Fisher \& Herr (2001);
d. Data mapping - Fisher \& Herr (2001);

4. (Re) Produção Algorítmica (Design Evolucionário) Algorithmic (re-)production (Evolutionary Design)

a. Algoritmos Genéticos (Genetic Algorithms) - Gero \& Kazakov (2001); Caldas (2008); Troiano \& Birtolo (2014);

b. Selective Procedures - Fisher \& Herr (2001); 
Figura 2: Visualização integrada de técnicas aplicáveis ao Design Generativo na perspectiva de Fisher e Herr (2001) e Singh \& Gu (2012), elaborado pelo autor.

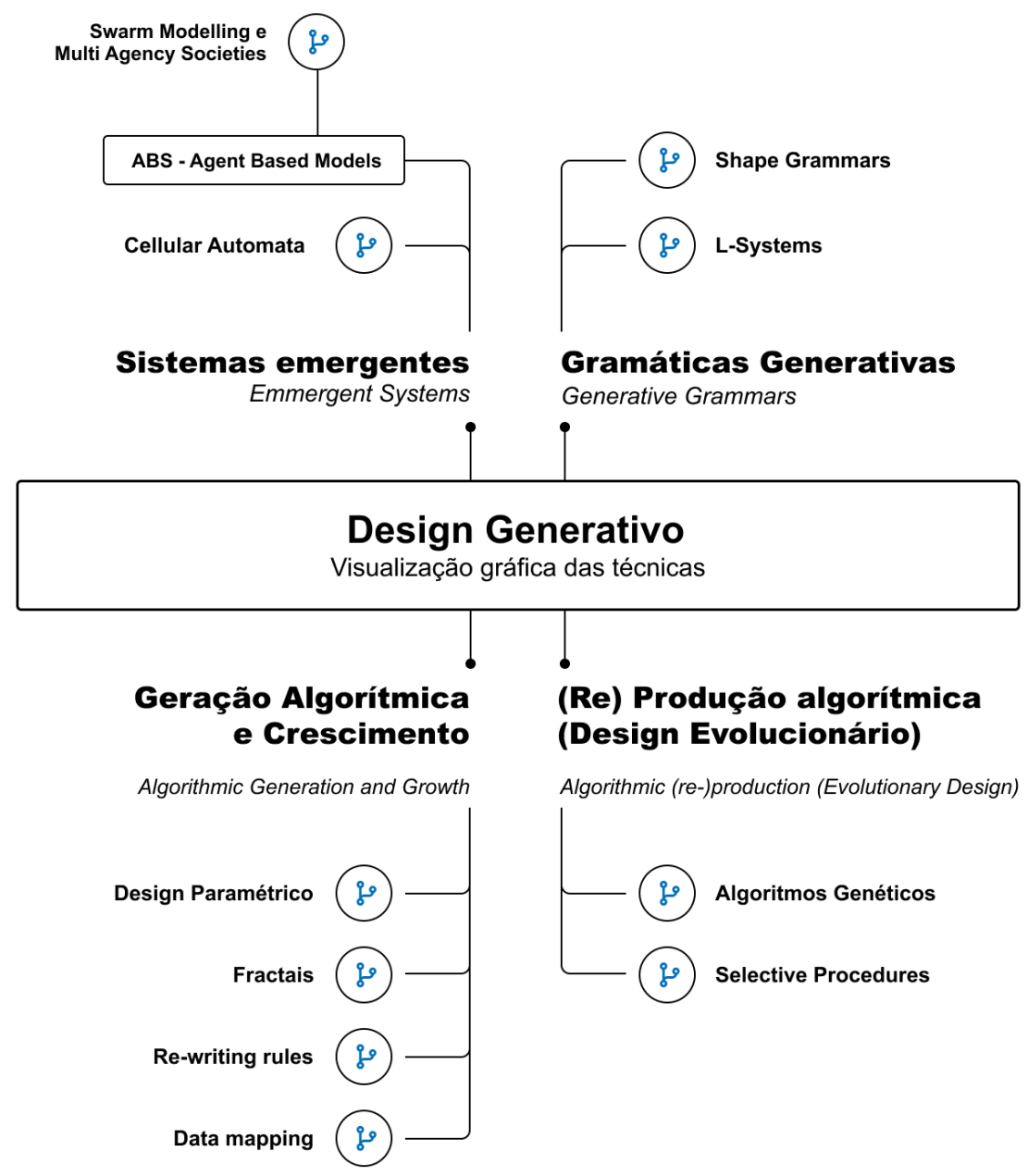

Vale reforçar que grande parte das metodologias apontadas tem origem no campo da Matemática (Fisher e Herr, 2001), o que reforça uma colaboração cada vez maior dessa disciplina e o Design. Considerando que as técnicas apresentadas na Figura 2 estão dispostas com relação a similaridades metodológicas, existem outras formas de classificação, como é o caso dos modelos linguísticos e biológicos. Tais classificações partem da ótica de como as regras são estabelecidas e oferecem uma alternativa à compreensão do contexto das técnicas no campo.

\section{Os modelos linguísticos e biológicos no Design Generativo}

Sistemas Generativos, em sua maioria, são sistemas computacionais que, por natureza, trabalham por conjuntos de regras simbólicas que processam, interpretam e produzem novos símbolos. Tais regras, quando programadas, podem revelar as ações de quem as constrói. 
Segundo Arida (2004, p.49), nos sistemas Generativos pode-se identificar dois modelos principais de construções dessas regras: os linguísticos e os biológicos. Quanto ao objeto, o primeiro estrutura-se direcionado à forma, enquanto o segundo ao ambiente.

Os modelos linguísticos estruturam-se com foco nas formas, que podem ser modificadas a partir de regras aplicadas diretamente a elas. Toma-se como exemplo as Gramáticas da Forma (Shape Grammars), que atuam como um conjunto de regras que podem ser aplicadas em uma forma inicial para gerar um conjunto de designs possíveis (Arida, 2004, p.61). Hwang (2017) exemplifica seu uso nas "cidades infinitas", modelagens de cidades em computação gráfica, como as realizadas no filme BIG HERO 6 (DISNEY, 2014) ${ }^{3}$, em que os módulos, como texturas, janelas e formas, são identificados, e as permutações entre eles geram um conjunto de opções, que no caso do filme foram utilizadas para formar cidades virtuais inteiras, otimizando tempo e investimentos.

Na Figura 3, percebe-se que inicialmente uma forma é escolhida e seus módulos identificados. Isso permite que a gramática da forma seja revelada, fazendo com que o sistema entre em ação, gerando permutações entre os módulos. Soluções desfavoráveis podem ser descartadas, e o sistema passa a trabalhar nos modelos escolhidos;

Figura 3: Esquema ilustrativo de exemplo de modelo linguístico no Design Generativo (Gramáticas da Forma). Elaborado pelo autor a partir da visão de Arida (2004).

\section{Modelo Linguístico (Gramáticas da Forma) | Design Generativo}

1.

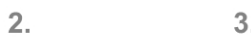

3.

4.

5.

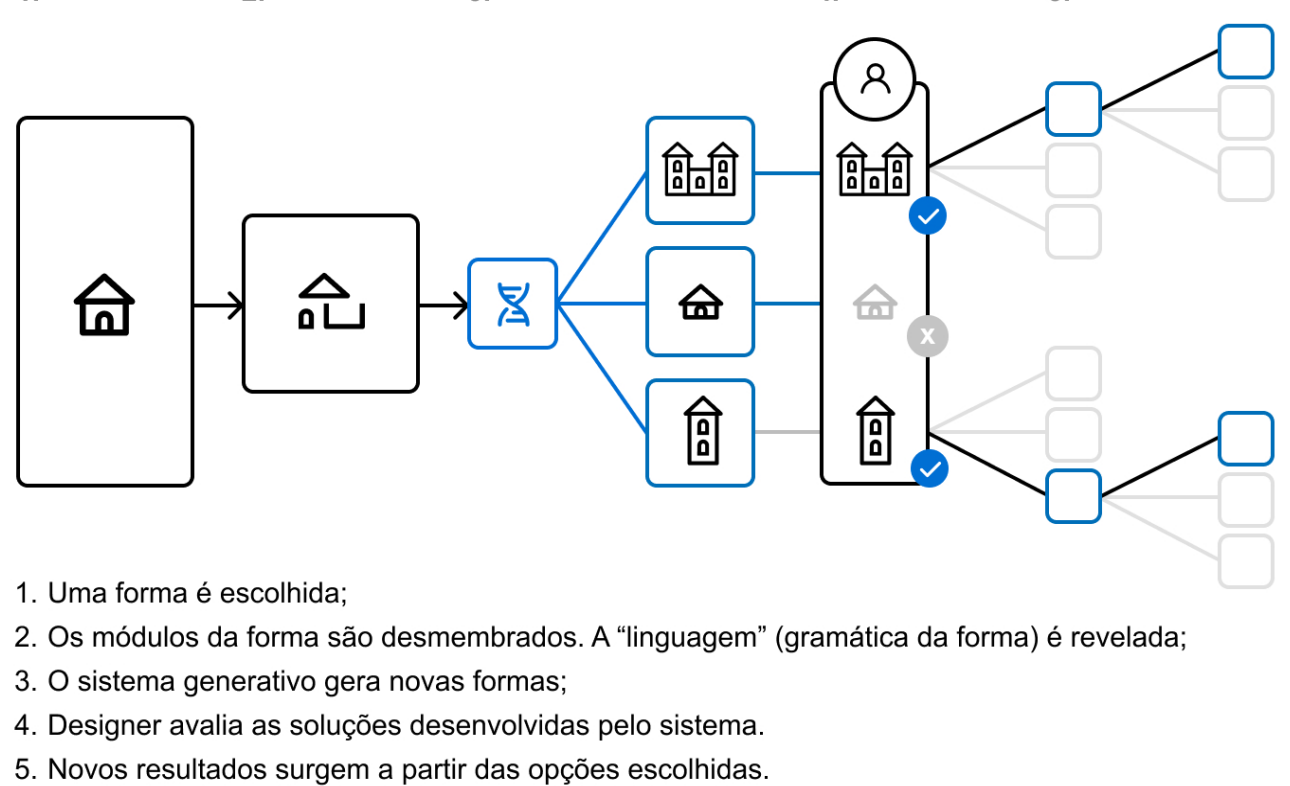

Já nos modelos biológicos, configura-se o ambiente, estabelecendo as regras e as forças presentes nele. A forma surge como resultado do contexto, em uma espécie de equilíbrio metabólico, em que a forma resultante alinha-se com todas as regras estabelecidas no

\footnotetext{
${ }^{2}$ Disponível em: <https://www.youtube.com/watch?v=t-VUpX-xVo4\&t=375s>

${ }^{3}$ Disponível em: <https://www.youtube.com/watch?v=40MqveftZzs>
} 
ambiente. Para modificar a forma, o designer precisa alterar as regras, gerando um novo estado de equilíbrio. Modelos biológicos podem ser estruturados a partir de algoritmos genéticos de base evolutiva que permitem que as formas evoluam em um ambiente virtual e possam ser selecionadas com critérios de seleção natural, ou seja, há a escolha das soluções mais adaptadas dentro de uma variedade de mutações.

Figura 4: Esquema ilustrativo de exemplo de modelo biológico no Design Generativo.

Elaborado pelo autor a partir da visão de Arida (2004);

\section{Biológico | Design Generativo}

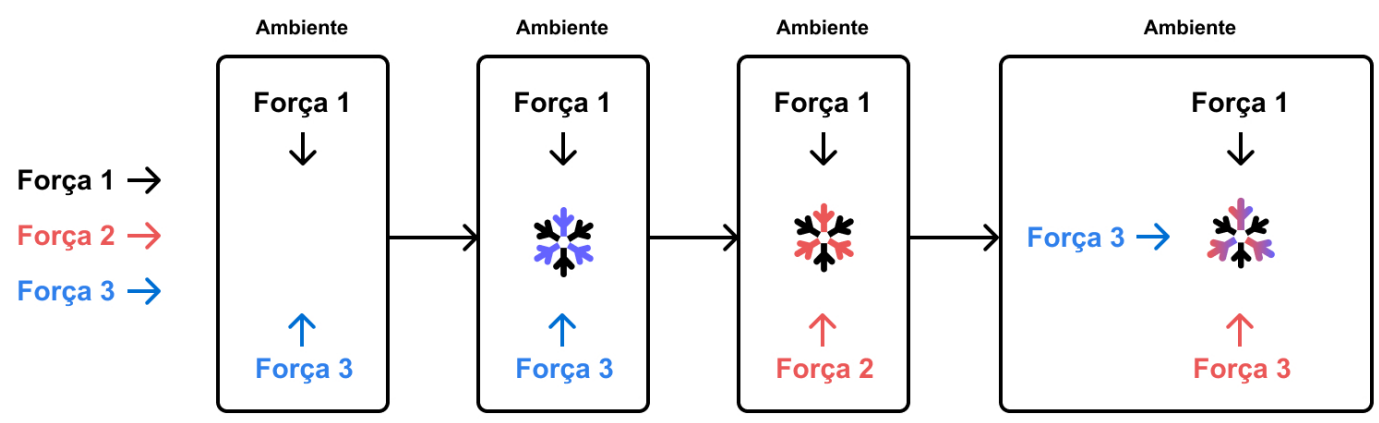

1. Regras e forças são designadas de acordo com o problema de design;

2. Conjunto de regras e forças gera um dado ambiente (contexto);

3. A forma resultante é fruto do equilíbrio entre as regras e forças do ambiente;

4. A substituição de novas regras e forças gera novas formas;

5. Forma final satisfaz o conjunto de regras estabelecidas no ambiente.

Seja por modelos linguísticos, biológicos ou por agrupamentos de técnicas por similaridades nas metodologias, como no dendograma apresentado na Figura 2, o presente artigo tem apresentado algumas formas de classificar as técnicas do Design Generativo. No entanto, somente a classificação não é suficiente para começarmos a enxergar padrões existentes nesse contexto. Estabelecer formas de monitoramentos e visualizações dessas técnicas é uma demanda que pode materializar-se a partir da aproximação com o Design da Informação.

\section{O Design da Informação como ferramenta de monitoramento de tecnologias e marcos do Design Generativo}

Em um contexto de aceleração técnica, visualizar dados complexos é tarefa essencial para que riscos e oportunidades sejam antecipados, dando à comunidade do Design mais poder decisório. Tal contexto assemelha-se ao que Flusser (2012, p.17) entende por manipulação, que é a capacidade de moldar certas situações abstratas, tornando-as palpáveis, o que permite aos seres humanos explicá-las. A partir dessa perspectiva, compreende-se que o campo do Design da Informação oferece recursos para uma abordagem sobre a complexidade presente no campo do Design Generativo, facilitando a sua análise. 
Em experiências como o Al Watch (Martinez-Plumed \& Hernandez-Orallo, 2020), organização dedicada a monitorar e debater os avanços da Inteligência Artificial (IA) na Europa, pesquisadores, empresas e organizações atuam conjuntamente para moldar o desenvolvimento técnico, propondo debates e reflexões. Como parte da experiência, a instituição oferece dashboards interativos para a consulta aos dados coletados, como no caso do Al Watch Landscape ${ }^{4}$, do Al Collaboratory ${ }^{5}$ e do Al History Timeline ${ }^{6}$, além de realizar publicações utilizando metodologias de monitoramento tecnológico, como é o caso das TRLS (Technology Readiness Levels), que permitem enxergar em que estágio cada técnica está na perspectiva de especialistas no tema (Martinez-Plumed \& Hernandez-Orallo, 2020).

Referências como essas podem inspirar a comunidade do Design Generativo a construir visualizações gráficas e monitoramentos técnicos de uma forma similar ao proposto no $\mathrm{Al}$ Watch, visto que em contextos como esses, de ampla evolução, as habilidades humanas de análise alcançam mais performance se apoiadas por sistemas computacionais que ajudem a enxergar mais profundamente o contexto.

Pretende-se, portanto, identificar alguns recursos de visualizações gráficas que podem permitir a análise das evoluções técnicas existentes no Design Generativo, como um primeiro passo para a criação de uma plataforma de monitoramento e consulta para a comunidade.

\section{Sistemas de informação: justificativas para o monitoramento das técnicas de Design Generativo}

Como disciplina formada por um conjunto de técnicas, em sua maioria aplicadas a sistemas computacionais, o Design Generativo pode beneficiar-se de um monitoramento a partir de visualizações de dados que nos apontem como tais técnicas têm sido utilizadas, seja de forma individualizada ou interconectada.

Segundo Meirelles (2013), o Design da Informação é o conjunto de práticas cujo principal propósito é informar, e as visualizações de dados e informações são comumente utilizadas no contexto de representações visuais processadas por computadores que amplificam a cognição e a compreensão de dados abstratos. Unifica-se então essa visão, do Design da Informação, em conjunto com Sighn \& Gu (2012), que investigaram a utilização de técnicas de Design Generativo a fim de que tenhamos recursos para propor visualizações para o monitoramento das tecnologias no campo.

Sighn (2011) entende que a utilização de somente uma técnica de Design Generativo pode gerar vieses e propõe um conhecimento integrado para escolha das técnicas mais recomendadas a um certo problema, permitindo que o designer interaja com mais de uma ao longo do seu processo criativo. Seu modelo estabelece quatro regras com funções específicas que ajudarão o designer a identificar as melhores técnicas, os melhores momentos para o

\footnotetext{
${ }^{4}$ Disponível em: <https://web.jrc.ec.europa.eu/dashboard/AI_WATCH_LANDSCAPE/index.html?bookmark= overview\&rdr=1626346417513>

${ }^{5}$ Disponível em: <https://knowledge4policy.ec.europa.eu/ai-watch/ai-collaboratory-tracking-evolution-ai_en >

${ }^{6}$ Disponível em: <https://knowledge4policy.ec.europa.eu/visualisation/ai-history-timeline_en >
} 
intercâmbio entre elas, como traduzi-las e como integrar todas as alternativas anteriores de forma sistematizada e planejada, que podem ser consultadas na Tabela 1.

Tabela 1: Framework integrado de Design Generativo (Singh \& Gu, 2012) - Adaptado e traduzido pelo autor.

\begin{tabular}{lll}
\hline Regras & Problema que resolve & Soluções \\
\hline Seleção & $\begin{array}{l}\text { Identificação da técnica recomendada ao } \\
\text { problema }\end{array}$ & Compatibilidades técnicas \\
\hline Gatilhos & $\begin{array}{l}\text { Momentos em que o designer pode } \\
\text { considerar a mudança de técnicas }\end{array}$ & $\begin{array}{l}\text { Regras que podem ser administradas em } \\
\text { intervalos regulares no desenvolvimento } \\
\text { de design como parte do processo de } \\
\text { avaliação }\end{array}$ \\
\hline Tradução & $\begin{array}{l}\text { Como os elementos identificados em } \\
\text { uma técnica podem ser traduzidos como } \\
\text { inputs para outras técnicas de Design } \\
\text { Generativo }\end{array}$ & $\begin{array}{l}\text { Intercâmbio - Como interconectar as } \\
\text { técnicas e fazer com que elas } \\
\text { conversem entre si }\end{array}$ \\
\hline Estratégia & $\begin{array}{l}\text { Como integrar as abordagens de baixo } \\
\text { para cima e de cima para baixo }\end{array}$ & $\begin{array}{l}\text { Em alguns casos é a integração das } \\
\text { regras de seleção, gatilhos e tradução } \\
\text { (3 anteriores) }\end{array}$ \\
\hline
\end{tabular}

Para o autor, o intercâmbio entre as técnicas gera problemas, mas também soluções possíveis. Entre os problemas destaca-se a necessidade de identificação da técnica mais alinhada ao problema. A partir das classificações de Meirelles (2013), propõem-se três visualizações possíveis unificadas em um sistema computacional, uma para cada regra, como pode ser visualizado na tabela 2 .

Tabela 2: Propostas de visualizações baseadas no framework integrado de Design Generativo de Sign (2011) - Elaborado pelo Autor.

\begin{tabular}{ll}
\hline Regras & Visualizações propostas \\
\hline Seleção & Direcionados pela Força (Modelos de Rede) e Nuvens de Palavras \\
\hline Gatilhos & Diagramas de Sankey (Modelos de Rede) \\
\hline Tradução & Diagramas de Sankey (Modelos de Rede) \\
\hline Estratégia & Sistema computacional que apresente as visualizações propostas nos tópicos anteriores \\
\hline
\end{tabular}

Como apresentado na Tabela 2, propõem-se visualizações, cada uma considerando aspectos de cada regra. Justificam-se as visualizações e interações sugeridas para cada etapa:

- Seleção - trata-se da necessidade de escolher a técnica mais recomendada de acordo com o problema de design (Sign, 2011). Partindo da definição, podemos compreender dois pontos importantes, o primeiro, de computar os problemas $(P)$, e o segundo, de 
monitorar a frequência de técnicas associadas a tais problemas. Técnicas $(T)$ associadas com maior frequência a um dado problema devem destacar-se na visualização. Sugere-se também que as técnicas devam ser estruturadas de modo fixo, para que não haja registros repetidos desnecessariamente. A visualização que pode funcionar é a de Modelos de Rede Direcionados pela Força (Meirelles, 2013, p.62), em que no ponto central há o problema, e nas conexões representam-se as técnicas, como demonstrado na figura 5. Para viabilizar a categorização, sugere-se que cada usuário registre o seu problema de modo livre, porém sempre vinculando a um problema macro, proposto pelo sistema. Ao clicar em um dado problema, o usuário pode acessar uma tabela com os percentuais de cada técnica, as documentações e criações realizadas, bem como uma nuvem de palavras que auxilie no processo de seleção;

Figura 5: Modelo de rede proposto pelo autor para a regra de Seleção.

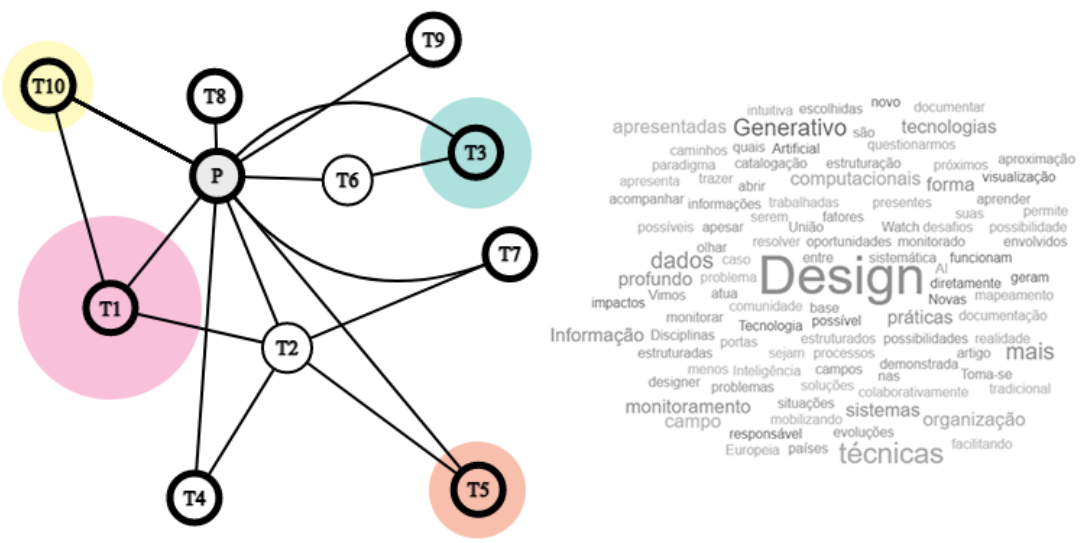

- Tradução e Gatilhos - Singh (2011) formula a necessidade de identificarmos as formas mais apropriadas de intercâmbio entre técnicas, o que nos revela a necessidade de documentar também suas formas de integração. Infere-se que podem existir modos diferentes de realizar a mesma integração, inclusive outros com alta similaridade entre si. Portanto, para não inviabilizar a visualização pela quantidade de variáveis, as conexões entre técnicas podem ser interativas, permitindo o clique para visualizar todo o acervo de documentação específica da integração em questão. Recomenda-se a utilização de Diagramas de Sankey (Meirelles, 2013, p.62), que orientam caminhos e interconexões entre pontos, destacando aqueles com maior volume. Isso permitirá que o designer consulte sempre que desejar migrar de tecnologias, bem como as planeje de modo antecipado, o que permite resolver a questão dos gatilhos, proposta por Singh. 
Figura 6: Diagrama de Sankey proposto pelo autor para o monitoramento de Traduções e Gatilhos entre as técnicas de Design Generativo.

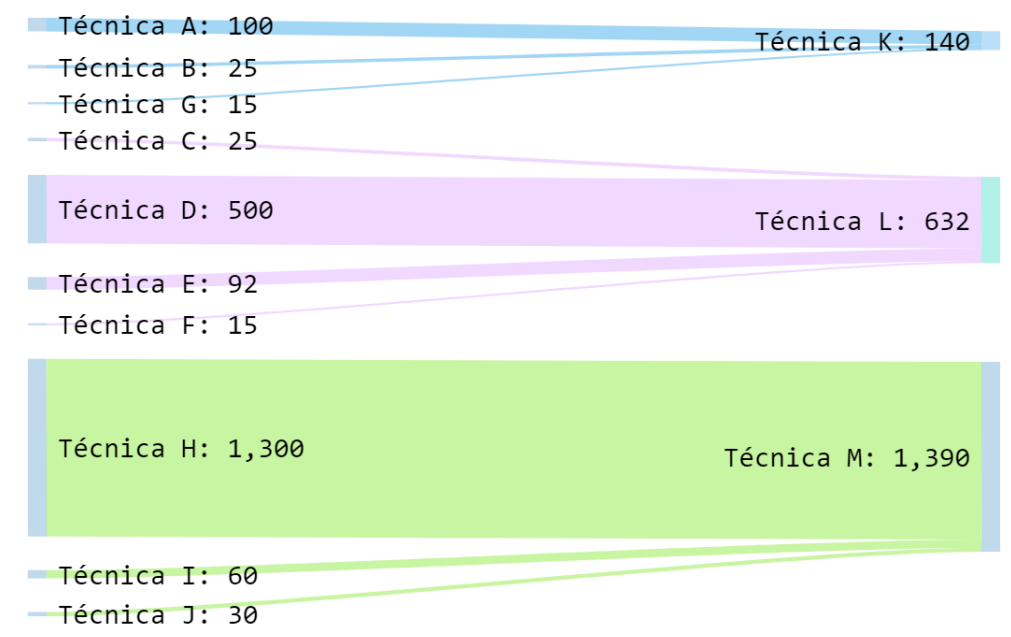

No modelo acima, o usuário pode estabelecer relações e filtros entre as técnicas, e o sistema processa o número de registros das conexões, dando relevância para os que possuem maior volume.

- Estratégia - Para resolver o quesito da estratégia (Sigh 2011), demanda-se a unificação das três etapas, facilitando o intercâmbio e a consulta à base de dados. A unificação das visualizações de dados previamente apresentadas em uma plataforma integrada pode dar recursos para que o designer navegue nas soluções relacionadas com o seu problema de design.

Além do intercâmbio proposto entre técnicas, entende-se que o Design Generativo pode apresentar potencial de documentação de processos e dados superior a outras áreas do Design, especialmente por valer-se da utilização de algoritmos e sistemas computacionais em mais etapas. No entanto, somente com um volume significativo de dados coletados é que teremos a capacidade de enxergar as mudanças e desenvolvimentos de caráter técnico com mais agilidade. Propõe-se, então, que o designer, ao interagir com as técnicas de Design Generativo, beneficie-se de uma comunidade organizada em torno de um sistema computacional. Tal sistema, ao permitir o compartilhamento de criações, poderá gerar um conjunto de dados que, ao serem dispostos em visualizações gráficas, passarão a ofertar insights para o designer, além de dar uma visão sistêmica de como o campo tem se desenvolvido tecnicamente. Tal escolha pelo sistema justifica-se também pela aceleração técnica que vivemos, sendo uma forma de monitoramento escalável.

Outras formas de visualizações também podem ser utilizadas de modo complementar às propostas, como é o caso dos infográficos, tema abordado na seção a seguir. 


\section{Infográficos como narrativa informacional}

Paralelamente à solução proposta, entende-se que a experiência das visualizações sugeridas assume um caráter mais exploratório do que informacional. Visando otimizar o acesso ao tema, propõe-se a criação de infográficos que atraiam os usuários às análises e abordem os temas a partir da transmissão da informação.

Na perspectiva de Meirelles (2013), infográficos são "visualizações formadas por aspectos gráficos, como ilustrações, símbolos, mapas e diagramas, que combinadas com a linguagem verbal transmitem uma informação que não seria possível de outra forma" (p.11). Já Krum (2014, p.6) os define reforçando a importância da narrativa, ao contar uma história completa a partir de um design que combine dados, visualizações, ilustrações, textos e imagens.

Krum (2014, p.58) apresenta a possibilidade de uma navegação horizontal ou vertical em infográficos disponibilizados online. Como há a visão de que os infográficos sejam hospedados em um site, é esperado que a navegação verticalizada tenda a ser mais facilmente adaptada para dispositivos mobile, o que pode gerar uma melhor navegação. Krum (2014, p.89) classifica os infográficos online em seis categorias (traduzido pelo autor):

1. Informativos - oferecem informações valiosas;

2. Persuasivos - possuem foco na "chamada para ação";

3. Explicações visuais - explicam ideias, conceitos ou processos, sem a necessidade de um conjunto de estatísticas, números ou dados;

4. Publicitários - focados em convencer a respeito de alguma aquisição ou conversão;

5. Relações públicas - promovem valor aos stakeholders ou dão visibilidade institucional a produtos e marcas;

6. Posters - design e venda de posters com infográficos.

Diante da possibilidade de utilização dos infográficos como recurso narrativo de informação para atrair usuários para uma segunda experiência, mais exploratória, no sistema computacional proposto na seção anterior, entende-se que seria possível utilizar todas as classificações propostas por Krum em infográficos pensados caso a caso. No entanto, como ponto de partida, optamos por propor um infográfico na perspectiva de "explicações visuais", cujo objeto é o conteúdo do próprio artigo em questão.

Utiliza-se o próprio conteúdo do presente artigo como forma de guiar o usuário por experiências complementares dentro da mesma informação, seja por um acesso que aconteça inicialmente pelo artigo e depois parta para o infográfico, ou no sentido inverso, o que pode otimizar a sua experiência com a pesquisa realizada.

Estrutura-se o conteúdo em cinco diferentes momentos: i) Ambientação sobre o Design Generativo; ii) Expectativas de mudanças; iii) Técnicas de Design Generativo; iv) Sistemas linguísticos e biológicos; v) A aproximação com o Design da Informação. 
Figura 7: Infográfico desenvolvido pelo autor - Disponível em: <https://bit.ly/3x2nKXF>
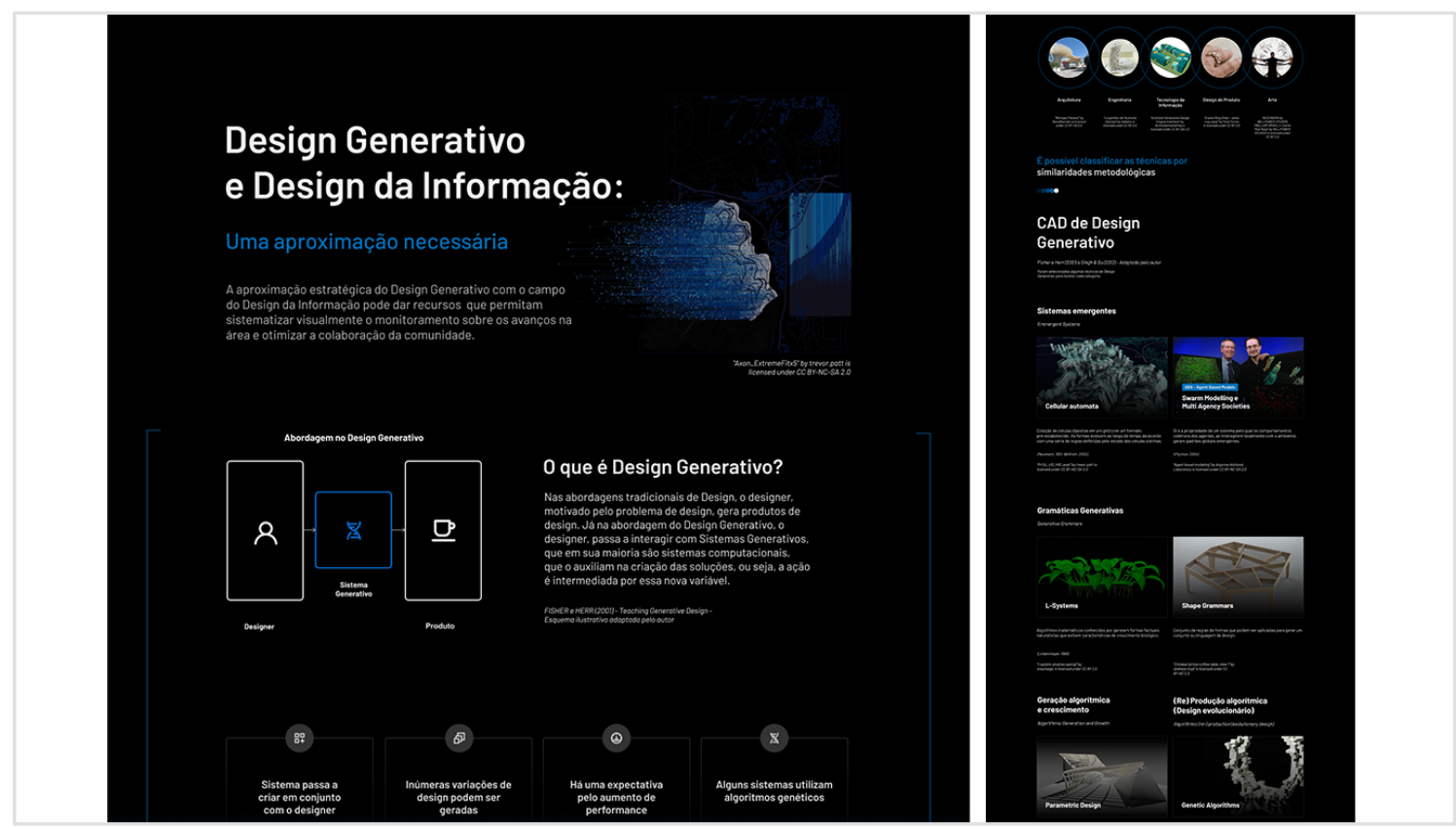

Como disciplinas, o Design Generativo e o Design da Informação têm muito a contribuir, e isso não se resume às visualizações propostas no presente artigo. As possibilidades são extensas e podem acontecer tanto na perspectiva técnica quanto nas reflexões sobre o próprio desmembramento dos campos. Na perspectiva adotada, constrói-se o argumento a partir de um olhar sobre as técnicas do Design Generativo e seu monitoramento, para que em momentos seguintes haja debates a respeito de seus impactos.

De todo modo, entende-se que o Design da Informação nos dá recursos para tangibilizar cenários, facilitando o processo decisório, e que o Design Generativo pode beneficiar-se dessa aproximação, a partir da análise de seus desmembramentos.

\section{Considerações finais}

A aproximação entre o Design Generativo e o Design da Informação amplia as possibilidades de um olhar aprofundado sobre as técnicas e tecnologias no Design. Disciplinas como o Design Generativo podem trazer práticas de documentação, presentes na Tecnologia da Informação, para as práticas do Design, o que pode permitir um monitoramento técnico maior, mobilizando colaboração e facilitando a estruturação de dados.

A partir dessa visão, o presente artigo realizou um breve apanhado do Design Generativo, reforçando o papel dos Sistemas Generativos, bem como a identificação de um cenário cada vez mais favorável ao campo pelo barateamento de soluções disponíveis na Nuvem e Aprendizado de Máquina, o que pode fazer florescer todo um contexto de disseminação dessas tecnologias no nosso dia a dia.

Como desafio, há a necessidade de monitorar o avanço técnico, cada vez mais acelerado, para que haja debates e reflexões sobre os eventuais impactos dessas transformações. 
Identificou-se aí a possibilidade de mitigar uma possível lacuna de categorização de técnicas, propondo um ponto de partida para um sistema computacional que atue de forma integrada e com amplo acesso a toda comunidade.

Fisher \& Herr (2001) e Singh \& Gu (2012) permitiram um olhar sobre uma possível categorização de técnicas por similaridade metodológica. De outro modo, a contribuição de Arida (2004) nos apresentou a classificação por tipos de regras, por meio dos modelos linguísticos e biológicos. Tais contribuições nos permitem um olhar mais sistemático, o que pode ser relevante para que a comunidade acesse tais técnicas com maior facilidade.

Conhecendo as dificuldades de acompanhar a evolução técnica, identificou-se a possibilidade de uma aproximação com o Design da Informação, muito inspirada pela experiência vivenciada no AI Watch, a partir da unificação de esforços da comunidade do Design Generativo em prol da colaboração e debate sobre as tecnologias.

Desse modo, partiu-se do framework de Singh \& Gu (2012) como ponto de partida para um intercâmbio e integração entre as técnicas. Para cada uma das regras, sugeriu-se um conjunto de visualizações gráficas estruturadas na visão de Meirelles (2013) que possam ser organizadas em um sistema computacional integrador que permita a interação de modo exploratório. Junto a isso, propõe-se a aplicação de infográficos como mídia de suporte para a apresentação de informações de um ponto de vista mais narrativo, atraindo os usuários para a utilização do sistema computacional.

$\mathrm{O}$ artigo mostrou que há a possibilidade de um mapeamento mais profundo sobre o campo das técnicas no Design Generativo e também de questionarmos quais técnicas apresentadas hoje podem ter seus dados estruturados. Novas situações geram novos problemas, e o Design possivelmente está diante de um novo paradigma, que apresenta desafios e oportunidades.

\section{Referencial Bibliográfico}

Arida, S. (2004, 5 19). Contextualizing Generative Design. Massachusetts Institute of Technology. https://dspace.mit.edu/handle/1721.1/17917?show=full

Caldas, L. (2008). Generation of energy-efficient architecture solutions applying GENE_ARCH: An evolution-based generative design system. Advanced Engineering Informatics, 22, 59-70. Science Direct.

Carley, K. (1994). Sociology: computational organization theory. Social Science Computer Review, (12), 611 e 624.

Ediz, Ö., \& Çaðdap, G. (2007). A computational architectural design model based on fractals. Open House International, 32(2).

Fisher, T., \& Herr, C. M. (2001). Teaching Generative Design. Semantic Scholar. Retrieved 04 19, 2021, from https://www.semanticscholar.org/paper/Teaching-Generative-Design-FischerHerr/ece74fd51aa94be7442ff30775d190a6756be5cd\#citing-papers

Flusser, V. (2012). O universo das imagens técnicas - O elogio da superficialidade. Anna Blume. 
Follet, J. (2020, 11 12). Al, Architecture, and Generative Design. Towards Data Science. Retrieved 04 19, 2021, from https://towardsdatascience.com/ai-architecture-and-generativedesign-e22320828d46

Frazer, J. (2016). Parametric Computation: History and Future. Architectural Design, 86, 18-23. https://doi.org/10.1002/ad.2019

Gero, J. S., \& Kazakov, V. (2001). A Genetic Engineering Approach to Genetic Algorithms. Evolutionary Computation, 12, 71-92. http://dx.doi.org/10.1162/10636560151075121.

Grimm, V., Revilla, E., Berger, U., Jeltsch, F., Mooij, W. M., Railsback, S. F., Thulke, H.-H., Weiner, J., Wiegand, T., \& DeAngelis, D. L. (2005). Pattern-oriented modeling of agentbased complex systems: Lessons from ecology. Science, 310, 987-991.

Harvard Business Review Analytic Services. (2018, 6 4). The Next Wave of Intelligent Design Automation. Harvard Business Review Analytic Services. Retrieved 04 19, 2021, from https://hbr.org/sponsored/2018/06/the-next-wave-of-intelligent-design-automation

Hwang, R. (2017). SHAPE GRAMMARS - Procedural generation techniques for virtual cities. Retrieved 04 19, 2021, from https://www.youtube.com/watch?v=t-VUpX-xVo4\&t=375s

Krum, R. (2014). Cool Infographics: Effective Communication with Data Visualization and Design. Wiley.

Lindenmayer, A. (1968). Mathematical models for cellular interaction in development. Journal of Theoretical Biology, 18, 280-289.

Macy, M. W., \& Willer, R. (2002). From factors to Actors: computational Sociology and agentbased modeling. Annual Review of Sociology, 28, 143 e 166.

Martinez-Plumed, F., \& Hernandez-Orallo, J. (2020). Al Watch: Methodology to Monitor the Evolution of AI Technologies. JRC Technical Report. 10.2760/643950

Mckinsey Global Institute. (2018, 05 23). Skill shift: Automation and the future of the workforce. Mckinsey \& Company. Retrieved 04 19, 2021, from https://www.mckinsey.com/featuredinsights/future-of-work/skill-shift-automation-and-the-future-of-the-workforce\#

Meirelles, I. (2013). Design for information: An introduction to the histories, theories, and best practices behind effective information visualizations. Rockport Publishers.

NASA Ames Research Center \& Bluck, J. (2004, 6 14). NASA.gov. NASA 'EVOLUTIONARY' SOFTWARE AUTOMATICALLY DESIGNS ANTENNA. Retrieved 04 19, 2021, from https://www.nasa.gov/centers/ames/news/releases/2004/04_55AR.html

Prusinkiewicz, P., \& Lindenmayer, A. (1991). The Algorithmic Beauty of Plants. Springer Verlag.

Rosa, H. (2014, 04 14). Entrevista especial: Hartmund Rosa. Diário de Pernambuco Modernizações Ambivalentes.

http://www.ccba.org.br/modernizacoesambivalentes/?tag=hartmut-rosa

Singh, V., \& Gu, N. $(2012,3)$. Towards an integrated generative design framework. Design Studies, 33, 185-207. Science Direct. https://doi.org/10.1016/j.destud.2011.06.001

Stiny, G. (1975). Pictorial and Formal Aspects of Shape and Shape Grammars. Birkhäuser Basel.

Stiny, G. (1980). Introduction to Shape and Shape Grammars. Environment and Planning B, 7(3), 343-351. 
Troiano, L., \& Birtolo, C. (2014). Genetic algorithms supporting generative design of user interfaces: Examples. Information Sciences, 259, 433-451.

von Newmann, J. (1951). The General and Logical Theory of Automata. 5(L.A. Jeffress), 1-41. Wolfram, S. (2002). A New Kind of Science. Wolfram Media.

\section{Sobre os autores}

Matheus Dantas de Moraes Almeida, Publicitário, formado pela Faculdade de Comunicação, Universidade de Brasília. Mestrando pelo Programa de Pós Graduação em Design (UnB) <matheusdmalmeida@gmail.com>

Ana Carolina Kalume Maranhão. Doutora em Comunicação. Professora da Faculdade de Comunicação da Universidade de Brasília e professora colaboradora do Programa de PósGraduação em Design da Universidade de Brasília. <ckalume@gmail.com> 